\title{
Topological Properties of Strong Solutions for the 3D Navier-Stokes Equations
}

\author{
Pavlo O. Kasyanov ${ }^{\mathrm{a}}$, Luisa Toscano ${ }^{\mathrm{b}}$, Nina V. Zadoianchuk ${ }^{\mathrm{a}}$ \\ ${ }^{a}$ Institute for Applied System Analysis, National Technical University of Ukraine "Kyiv \\ Polytechnic Institute", Peremogy ave., 37, build, 35, 03056, Kyiv, Ukraine, \\ kasyanov@i.ua,ninellll@i.ua. \\ ${ }^{b}$ University of Naples "Federico II", Dep. Math. and Appl. R.Caccioppoli, via Claudio \\ 21,80125 Naples,Italy,luisatoscano@libero.it
}

\begin{abstract}
In this paper we give a criterion for the existence of global strong solutions for the 3D Navier-Stokes system for any regular initial data.
\end{abstract}

Keywords: 3D Navier-Stokes System, Strong Solution

\section{Introduction}

Let $\Omega \subseteq \mathbb{R}^{3}$ be a bounded open set with sufficiently smooth boundary $\partial \Omega$ and $0<T<+\infty$. We consider the incompressible Navier-Stokes equations

$$
\left\{\begin{array}{l}
y_{t}+(y \cdot \nabla) y=\nu \Delta y-\nabla p+f \text { in } Q=\Omega \times(0, T), \\
\operatorname{div} y=0 \text { in } Q, \\
y=0 \text { on } \partial \Omega \times(0, T), \quad y(x, 0)=y_{0}(x) \text { in } \Omega,
\end{array}\right.
$$

where $\nu>0$ is a constant. We define the usual function spaces

$$
\begin{gathered}
\mathcal{V}=\left\{u \in\left(C_{0}^{\infty}(\Omega)\right)^{3}: \operatorname{div} u=0\right\}, \\
H=\text { closure of } \mathcal{V} \text { in }\left(L^{2}(\Omega)\right)^{3}, \quad V=\left\{u \in\left(H_{0}^{1}(\Omega)\right)^{3}: \operatorname{div} u=0\right\} .
\end{gathered}
$$

We denote by $V^{*}$ the dual space of $V$. The spaces $H$ and $V$ are separable Hilbert spaces and $V \subset H \subset V^{*}$ with dense and compact embedding when $H$ is identified with its dual $H^{*}$. Let $(\cdot, \cdot),\|\cdot\|_{H}$ and $((\cdot, \cdot)),\|\cdot\|_{V}$ be the inner product and the norm in $H$ and $V$, respectively, and let $\langle\cdot, \cdot\rangle$ be the pairing between $V$ and $V^{*}$. For $u, v, w \in V$, the equality

$$
b(u, v, w)=\int_{\Omega} \sum_{i, j=1}^{3} u_{i} \frac{\partial v_{j}}{\partial x_{i}} w_{j} d x
$$


defines a trilinear continuous form on $V$ with $b(u, v, v)=0$ when $u \in V$ and $v \in$ $\left(H_{0}^{1}(\Omega)\right)^{3}$. For $u, v \in V$, let $B(u, v)$ be the element of $V^{*}$ defined by $\langle B(u, v), w\rangle=$ $b(u, v, w)$ for all $w \in V$.

We say that the function $y$ is a weak solution of $\operatorname{Pr}$. (1) on $[0, T]$, if $y \in$ $L^{\infty}(0, T ; H) \cap L^{2}(0, T ; V), \frac{d y}{d t} \in L^{1}\left(0, T ; V^{*}\right)$, if

$$
\frac{d}{d t}(y, v)+\nu((y, v))+b(y, y, v)=\langle f, v\rangle \quad \text { for all } v \in V,
$$

in the sense of distributions on $(0, T)$, and if $y$ satisfies the energy inequality

$$
V(y)(t) \leq V(y)(s) \text { for all } t \in[s, T],
$$

for a.e. $s \in(0, T)$ and for $s=0$, where

$$
V(y)(t):=\frac{1}{2}\|y(t)\|_{H}^{2}+\nu \int_{0}^{t}\|y(\tau)\|_{V}^{2} d \tau-\int_{0}^{t}\langle f(\tau), y(\tau)\rangle d \tau .
$$

This class of solutions is called Leray-Hopf or physical one. If $f \in L^{2}\left(0, T ; V^{*}\right)$, and if $y$ satisfies (2), then $y \in C\left([0, T] ; H_{w}\right), \frac{d y}{d t} \in L^{\frac{4}{3}}\left(0, T ; V^{*}\right)$, where $H_{w}$ denotes the space $H$ endowed with the weak topology. In particular, the initial condition $y(0)=y_{0}$ makes sense for any $y_{0} \in H$.

Let $A: V \rightarrow V^{*}$ be the linear operator associated to the bilinear form $((u, v))=$ $\langle A u, v\rangle$. Then $A$ is an isomorphism from $D(A)$ onto $H$ with $D(A)=\left(H^{2}(\Omega)\right)^{3} \cap V$. We recall that the embedding $D(A) \subset V$ is dense and continuous. Moreover, we assume $\|A u\|_{H}$ as the norm on $D(A)$, which is equivalent to the one induced by $\left(H^{2}(\Omega)\right)^{3}$. The Problem (11) can be rewritten as

$$
\left\{\begin{array}{l}
\frac{d y}{d t}+\nu A y+B(y, y)=f \text { in } V^{*} \\
y(0)=y_{0}
\end{array}\right.
$$

where the first equation we understand in the sense of distributions on $(0, T)$. Now we write

$$
\mathcal{D}\left(y_{0}, f\right)=\{y: y \text { is a weak solution of Pr. (11) on }[0, T]\} \text {. }
$$

It is well known (cf. [1] ) that if $f \in L^{2}\left(0, T ; V^{*}\right)$, and if $y_{0} \in H$, then $\mathcal{D}\left(y_{0}, f\right)$ is not empty.

A weak solution $y$ of $\operatorname{Pr}$. (11) on $[0, T]$ is called a strong one, if it additionally belongs to Serrin's class $L^{8}\left(0, T ;\left(L^{4}(\Omega)\right)^{3}\right)$. We note that any strong solution $y$ of Pr. (1) on $[0, T]$ belongs to $C([0, T] ; V) \cap L^{2}(0, T ; D(A))$ and $\frac{d y}{d t} \in L^{2}(0, T ; H)$ (cf. 2, Theorem 1.8.1, p. 296] and references therein). 
For any $f \in L^{\infty}(0, T ; H)$ and $y_{0} \in V$ it is well known the only local existence of strong solutions for the 3D Navier-Stokes equations (cf. [2, 1, 3] and references therein). Here we provide a criterion for existence of strong solutions for Pr. (11) on $[0, T]$ for any initial data $y_{0} \in V$ and $0<T<+\infty$.

\section{Topological Properties of Strong Solutions}

The main result of this note has the following form.

Theorem 2.1. Let $f \in L^{2}(0, T ; H)$ and $y_{0} \in V$. Then either for any $\lambda \in[0,1]$ there is an $y_{\lambda} \in C([0, T] ; V) \cap L^{2}(0, T ; D(A))$ such that $y_{\lambda} \in \mathcal{D}\left(\lambda y_{0}, \lambda f\right)$, or the set

$$
\left\{y \in C([0, T] ; V) \cap L^{2}(0, T ; D(A)): y \in \mathcal{D}\left(\lambda y_{0}, \lambda f\right), \lambda \in(0,1)\right\}
$$

is unbounded in $L^{8}\left(0, T ;\left(L^{4}(\Omega)\right)^{3}\right)$.

In the proof of Theorem 2.1 we use an auxiliary statement connected with continuity property of strong solutions on parameters of Pr. (1) in Serrin's class $L^{8}\left(0, T ;\left(L^{4}(\Omega)\right)^{3}\right)$.

Theorem 2.2. Let $f \in L^{2}(0, T ; H)$ and $y_{0} \in V$. If $y$ is a strong solution for $\operatorname{Pr}$. (11) on $[0, T]$, then there exist $L, \delta>0$ such that for any $z_{0} \in V$ and $g \in$ $L^{2}(0, T ; H)$, satisfying the inequality

$$
\left\|z_{0}-y_{0}\right\|_{V}^{2}+\|g-f\|_{L^{2}(0, T ; H)}^{2}<\delta
$$

the set $\mathcal{D}\left(z_{0}, g\right)$ is one-point set $\{z\}$ which belongs to $C([0, T] ; V) \cap L^{2}(0, T ; D(A))$, and

$$
\|z-y\|_{C([0, T] ; V)}^{2}+\frac{\nu}{2}\|z-y\|_{D(A)}^{2} \leq L\left(\left\|z_{0}-y_{0}\right\|_{V}^{2}+\|g-f\|_{L^{2}(0, T ; H)}^{2}\right) .
$$

Remark 2.3. We note that from Theorem 2.2 with $z_{0} \in V$ and $g \in L^{2}(0, T ; H)$ with $\left\|z_{0}\right\|_{V}^{2}+\|g\|_{L^{2}(0, T ; H)}^{2}$ sufficiently small, Problem (1) has only one global strong solution.

Remark 2.4. Theorem 2.2 provides that, if for any $\lambda \in[0,1]$ there is an $y_{\lambda} \in$ $L^{8}\left(0, T ;\left(L^{4}(\Omega)\right)^{3}\right)$ such that $y_{\lambda} \in \mathcal{D}\left(\lambda y_{0}, \lambda f\right)$, then the set

$$
\left\{y \in C([0, T] ; V) \cap L^{2}(0, T ; D(A)): y \in \mathcal{D}\left(\lambda y_{0}, \lambda f\right), \lambda \in(0,1)\right\}
$$

is bounded in $L^{8}\left(0, T ;\left(L^{4}(\Omega)\right)^{3}\right)$. 
If $\Omega$ is a $C^{\infty}$-domain and if $f \in C_{0}^{\infty}(\overline{(0, T) \times \Omega})^{3}$, then any strong solution $y$ of Pr. (1) on $[0, T]$ belongs to $C^{\infty}((0, T] \times \Omega)^{3}$ and $p \in C^{\infty}((0, T] \times \Omega)$ (cf. [2, Theorem 1.8.2, p. 300] and references therein). This fact directly provides the next corollary of Theorems 2.1 and 2.2 .

Corollary 2.5. Let $\Omega$ be a $C^{\infty}$-domain, $f \in C_{0}^{\infty}(\overline{(0, T) \times \Omega})^{3}$. Then either for any $y_{0} \in V$ there is a strong solution of $\operatorname{Pr}$. (1) on $[0, T]$, or the set

$$
\left\{y \in C^{\infty}((0, T] \times \Omega)^{3}: y \in \mathcal{D}\left(\lambda y_{0}, \lambda f\right), \lambda \in(0,1)\right\}
$$

is unbounded in $L^{8}\left(0, T ;\left(L^{4}(\Omega)\right)^{3}\right)$ for some $y_{0} \in C_{0}^{\infty}(\Omega)^{3}$.

\section{Proof of Theorem 2.2}

Let $f \in L^{2}(0, T ; H), y_{0} \in V$, and $y \in C([0, T] ; V) \cap L^{2}(0, T ; D(A))$ be a strong solution of Pr. (1) on $[0, T]$. Due to [4], [1, Chapter 3] the set $\mathcal{D}\left(y_{0}, f\right)=\{y\}$. Let us now fix $z_{0} \in V$ and $g \in L^{2}(0, T ; H)$ satisfying (7) with

$$
\delta=\min \left\{1 ; \frac{\nu}{4}\right\} e^{-2 T C}, C=\max \left\{\frac{27 c^{4}}{2 \nu^{3}} ; \frac{7^{8} c^{8}}{2^{12} \nu^{7}}\right\}\left(\|y\|_{C([0, T] ; V)}^{4}+1\right)^{2},
$$

$c>0$ is a constant from the inequalities (cf. [2, 1])

$$
\begin{array}{cl}
|b(u, v, w)| \leq c\|u\|_{V}\|v\|_{V}^{\frac{1}{2}}\|v\|_{D(A)}^{\frac{1}{2}}\|w\|_{H} \quad \forall u \in V, v \in D(A), w \in H ; \\
|b(u, v, w)| \leq c\|u\|_{D(A)}^{\frac{3}{4}}\|u\|_{V}^{\frac{1}{4}}\|v\|_{V}\|w\|_{H} \quad \forall u \in D(A), v \in V, w \in H .
\end{array}
$$

The auxiliary problem

$$
\left\{\begin{array}{l}
\frac{d \eta}{d t}+\nu A \eta+B(\eta, \eta)+B(y, \eta)+B(\eta, y)=g-f \text { in } V^{*} \\
\eta(0)=z_{0}-y_{0}
\end{array}\right.
$$

has a strong solution $\eta \in C([0, T] ; V) \cap L^{2}(0, T ; D(A))$ with $\frac{d \eta}{d t} \in L^{2}(0, T ; H)$, i.e.

$$
\frac{d}{d t}(\eta, v)+\nu((\eta, v))+b(\eta, \eta, v)+b(y, \eta, v)+b(\eta, y, v)=\langle g-f, v\rangle \quad \text { for all } v \in V,
$$

in the sense of distributions on $(0, T)$. In fact, let $\left\{w_{j}\right\}_{j \geq 1} \subset D(A)$ be a special basis (cf. [5, p. 56]), i.e. $A w_{j}=\lambda_{j} w_{j}, j=1,2, \ldots, 0<\lambda_{1} \leq \lambda_{2} \leq \ldots, \lambda_{j} \rightarrow+\infty$, $j \rightarrow+\infty$. We consider Galerkin approximations $\eta_{m}:[0, T] \rightarrow \operatorname{span}\left\{w_{j}\right\}_{j=1}^{m}$ for solutions of Pr. (12) satisfying

$$
\frac{d}{d t}\left(\eta_{m}, w_{j}\right)+\nu\left(\left(\eta_{m}, w_{j}\right)\right)+b\left(\eta_{m}, \eta_{m}, w_{j}\right)+b\left(y, \eta_{m}, w_{j}\right)+b\left(\eta_{m}, y, w_{j}\right)=\left\langle g-f, w_{j}\right\rangle,
$$


with $\left(\eta_{m}(0), w_{j}\right)=\left(z_{0}-y_{0}, w_{j}\right), j=\overline{1, m}$. Due to (10), (11) and Young's inequality we get

$$
\begin{gathered}
2\left\langle g-f, A \eta_{m}\right\rangle \leq 2\|g-f\|_{H}\left\|\eta_{m}\right\|_{D(A)} \leq \frac{\nu}{4}\left\|\eta_{m}\right\|_{D(A)}^{2}+\frac{4}{\nu}\|f-g\|_{H}^{2} ; \\
-2 b\left(\eta_{m}, \eta_{m}, A \eta_{m}\right) \leq 2 c\left\|\eta_{m}\right\|_{V}^{\frac{3}{2}}\left\|\eta_{m}\right\|_{D(A)}^{\frac{3}{2}} \leq \frac{\nu}{2}\left\|\eta_{m}\right\|_{D(A)}^{2}+\frac{27 c^{4}}{2 \nu^{3}}\left\|\eta_{m}\right\|_{V}^{6} ; \\
-2 b\left(y, \eta_{m}, A \eta_{m}\right) \leq 2 c\|y\|_{V}\left\|\eta_{m}\right\|_{V}^{\frac{1}{2}}\left\|\eta_{m}\right\|_{D(A)}^{\frac{3}{2}} \leq \frac{\nu}{2}\left\|\eta_{m}\right\|_{D(A)}^{2}+\frac{27 c^{4}}{2 \nu^{3}}\|y\|_{C([0, T] ; V)}^{4}\left\|\eta_{m}\right\|_{V}^{2} ; \\
-2 b\left(\eta_{m}, y, A \eta_{m}\right) \leq 2 c\left\|\eta_{m}\right\|_{D(A)}^{\frac{7}{4}}\left\|\eta_{m}\right\|_{V}^{\frac{1}{4}}\|y\|_{V} \leq \frac{\nu}{2}\left\|\eta_{m}\right\|_{D(A)}^{2}+\frac{7^{8} c^{8}}{2^{12} \nu^{7}}\|y\|_{C([0, T] ; V)}^{8}\left\|\eta_{m}\right\|_{V}^{2} .
\end{gathered}
$$

Thus,

$$
\frac{d}{d t}\left\|\eta_{m}\right\|_{V}^{2}+\frac{\nu}{4}\left\|\eta_{m}\right\|_{D(A)}^{2} \leq C\left(\left\|\eta_{m}\right\|_{V}^{2}+\left\|\eta_{m}\right\|_{V}^{6}\right)+\frac{4}{\nu}\|g-f\|_{H}^{2},
$$

where $C>0$ is a constant from (9). Hence, the absolutely continuous function $\varphi=\min \left\{\left\|\eta_{m}\right\|_{V}^{2}, 1\right\}$ satisfies the inequality $\frac{d}{d t} \varphi \leq 2 C \varphi+\frac{4}{\nu}\|g-f\|_{H}^{2}$, and therefore $\varphi \leq L\left(\left\|z_{0}-y_{0}\right\|_{V}^{2}+\|g-f\|_{L^{2}(0, T ; H)}^{2}\right)<1$ on $[0, T]$, where $L=\delta^{-1}$. Thus, $\left\{\eta_{n}\right\}_{n \geq 1}$ is bounded in $L^{\infty}(0, T ; V) \cap L^{2}(0, T ; D(A))$ and $\left\{\frac{d}{d t} \eta_{n}\right\}_{n \geq 1}$ is bounded in $L^{2}(0, T ; H)$. In a standard way we get that the limit function $\eta$ of $\eta_{n}, n \rightarrow+\infty$, is a strong solution of Pr. (12) on [0,T]. Due to [4], [1, Chapter 3] the set $\mathcal{D}\left(z_{0}, g\right)$ is onepoint $z=y+\eta \in L^{8}\left(0, T ;\left(L^{4}(\Omega)\right)^{3}\right)$. So, $z$ is strong solution of $\operatorname{Pr}$. (11) on $[0, T]$ satisfying (8).

The theorem is proved.

\section{Proof of Theorem 2.1}

We provide the proof of Theorem 2.1. Let $f \in L^{2}(0, T ; H)$ and $y_{0} \in V$. We consider the $3 \mathrm{D}$ controlled Navier-Stokes system (cf. [7, [6])

$$
\left\{\begin{array}{l}
\frac{d y}{d t}+\nu A y+B(z, y)=f \\
y(0)=y_{0}
\end{array}\right.
$$

where $z \in L^{8}\left(0, T ;\left(L^{4}(\Omega)\right)^{3}\right)$.

By using standard Galerkin approximations (see [1]) it is easy to show that for any $z \in L^{8}\left(0, T ;\left(L^{4}(\Omega)\right)^{3}\right)$ there exists an unique weak solution $y \in L^{\infty}(0, T ; H) \cap$ $L^{2}(0, T ; V)$ of $\operatorname{Pr}$. (13) on $[0, T]$, that is,

$$
\frac{d}{d t}(y, v)+\nu((y, v))+b(z, y, v)=\langle f, v\rangle, \text { for all } v \in V
$$


in the sense of distributions on $(0, T)$. Moreover, by the inequality

$$
|b(u, v, A v)| \leq c_{1}\|u\|_{\left(L^{4}(\Omega)\right)^{3}}\|v\|_{V}^{\frac{1}{4}}\|v\|_{D(A)}^{\frac{7}{4}} \leq \frac{\nu}{2}\|v\|_{D(A)}^{2}+c_{2}\|u\|_{\left(L^{4}(\Omega)\right)^{3}}^{8}\|v\|_{V}^{2},
$$

for all $u \in\left(L^{4}(\Omega)\right)^{3}$ and $v \in D(A)$, where $c_{1}, c_{2}>0$ are some constants that do not depend on $u, v$ (cf. [1] ), we find that $y \in C([0, T] ; V) \cap L^{2}(0, T ; D(A))$ and $B(z, y) \in L^{2}(0, T ; H)$, so $\frac{d y}{d t} \in L^{2}(0, T ; H)$ as well. We add that, for any $z \in$ $L^{8}\left(0, T ;\left(L^{4}(\Omega)\right)^{3}\right)$ and corresponding weak solution $y \in C([0, T] ; V) \cap L^{2}(0, T ; D(A))$ of (13) on $[0, T]$, by using Gronwall inequality, we obtain

$$
\begin{gathered}
\|y(t)\|_{V}^{2} \leq\left\|y_{0}\right\|_{V}^{2} e^{2 c_{2} \int_{0}^{t}\|z(t)\|_{\left(L^{4}(\Omega)\right)^{3}}^{8} d t}, \quad \forall t \in[0, T] ; \\
\nu \int_{0}^{T}\|y(t)\|_{D(A)}^{2} d t \leq\left\|y_{0}\right\|_{V}^{2}\left[1+2 c_{2} e^{2 c_{2} \int_{0}^{T}\|z(t)\|_{\left(L^{4}(\Omega)\right)^{3}}^{8} d t}\|z\|_{L^{8}\left(0, T ;\left(L^{4}(\Omega)\right)^{3}\right)}^{8}\right] .
\end{gathered}
$$

Let us consider the operator $F: L^{8}\left(0, T ;\left(L^{4}(\Omega)\right)^{3}\right) \rightarrow L^{8}\left(0, T ;\left(L^{4}(\Omega)\right)^{3}\right)$, where $F(z) \in C([0, T] ; V) \cap L^{2}(0, T ; D(A))$ is the unique weak solution of (13) on $[0, T]$ corresponded to $z \in L^{8}\left(0, T ;\left(L^{4}(\Omega)\right)^{3}\right)$.

Let us check that $F$ is a compact transformation of Banach space $L^{8}\left(0, T ;\left(L^{4}(\Omega)\right)^{3}\right)$ into itself (cf. [8]). In fact, if $\left\{z_{n}\right\}_{n \geq 1}$ is a bounded sequence in $L^{8}\left(0, T ;\left(L^{4}(\Omega)\right)^{3}\right)$, then, due to (15) and (16), the respective weak solutions $y_{n}, n=1,2, \ldots$, of Pr. (13) on $[0, T]$ are uniformly bounded in $C([0, T] ; V) \cap L^{2}(0, T ; D(A))$ and their time derivatives $\frac{d y_{n}}{d t}, n=1,2, \ldots$, are uniformly bounded in $L^{2}(0, T ; H)$. So, $\left\{F\left(z_{n}\right)\right\}_{n \geq 1}$ is a precompact set in $L^{8}\left(0, T ;\left(L^{4}(\Omega)\right)^{3}\right)$. In a standard way we deduce that $F: L^{8}\left(0, T ;\left(L^{4}(\Omega)\right)^{3}\right) \rightarrow L^{8}\left(0, T ;\left(L^{4}(\Omega)\right)^{3}\right)$ is continuous mapping.

Since $F$ is a compact transformation of $L^{8}\left(0, T ;\left(L^{4}(\Omega)\right)^{3}\right)$ into itself, Schaefer's Theorem (cf. [8, p. 133] and references therein) and Theorem 2.2 provide the statement of Theorem 2.1. We note that Theorem 2.2 implies that the set $\{z \in$ $\left.L^{8}\left(0, T ;\left(L^{4}(\Omega)\right)^{3}\right): z=\lambda F(z), \lambda \in(0,1)\right\}$ is bounded in $L^{8}\left(0, T ;\left(L^{4}(\Omega)\right)^{3}\right)$ iff the set defined in (6) is bounded in $L^{8}\left(0, T ;\left(L^{4}(\Omega)\right)^{3}\right)$.

The theorem is proved.

\section{References}

[1] R. Temam, Navier-Stokes equations, North-Holland, Amsterdam, 1979.

[2] H. Sohr, The Navier-Stokes Equations. An Elementary Functional Analytic Approach, Verlag, Birkhäuser, 2001.

[3] M.Z. Zgurovsky, P.O. Kasyanov, O.V. Kapustyan, J. Valero, N.V. Zadoianchuk, Evolution Inclusions and Variation Inequalities for Earth Data Processing III. Springer, Berlin, 2012. 
[4] J. Serrin, The initial value problem for the Navier-Stokes equations, in: R.E. Langer (editor), Nonlinear Problems, University of Wisconsin Press, Madison, 1963, pp. 69-98.

[5] R. Temam, Infinite-Dimensional Dynamical Systems in Mechanics and Physics, Springer-Verlag, New York, 1988.

[6] V.S. Melnik, L. Toscano, On weak extensions of extreme problems for nonlinear operator equations. Part I. Weak solutions, J. Automat. Inf. Scien. 38 (2006) 68-78.

[7] O.V. Kapustyan, P.O. Kasyanov, J. Valero, Pullback attractors for a class of extremal solutions of the 3D Navier-Stokes system, J. Math. Anal. Appl. 373 (2011) 535-547.

[8] J. Cronin, Fixed points and topological degree in nonlinear analysis, American Mathematical Society, Providence, RI, 1964. 